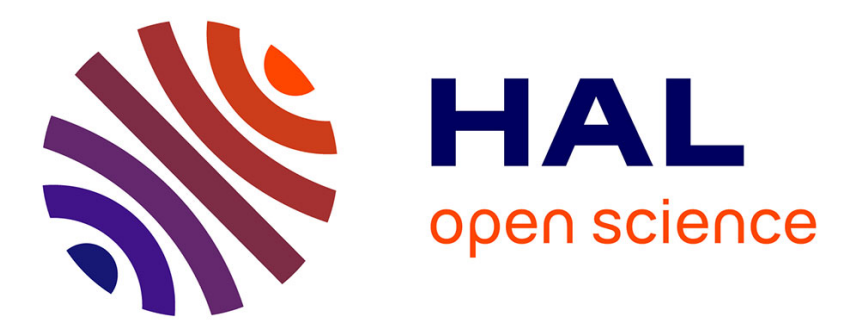

\title{
Influence of moisture content on reflectance of granular materials. Part II: optical measurements and modelling
}

Hélène Garay, Amandine Monnard, Dominique Lafon-Pham

\section{To cite this version:}

Hélène Garay, Amandine Monnard, Dominique Lafon-Pham. Influence of moisture content on reflectance of granular materials. Part II: optical measurements and modelling. Granular Matter, 2016, 18 (3), 10.1007/s10035-016-0649-6 . hal-02078364

\section{HAL Id: hal-02078364 \\ https://hal.science/hal-02078364}

Submitted on 20 Feb 2020

HAL is a multi-disciplinary open access archive for the deposit and dissemination of scientific research documents, whether they are published or not. The documents may come from teaching and research institutions in France or abroad, or from public or private research centers.
L'archive ouverte pluridisciplinaire HAL, est destinée au dépôt et à la diffusion de documents scientifiques de niveau recherche, publiés ou non, émanant des établissements d'enseignement et de recherche français ou étrangers, des laboratoires publics ou privés. 


\title{
Influence of moisture content on reflectance of granular materials. Part II: optical measurements and modelling
}

\author{
Helene Garay $^{1}$ • Amandine Monnard ${ }^{1}$ - Dominique Lafon-Pham ${ }^{1}$
}

\begin{abstract}
In some fields, it's important to control the moisture content of granular materials without any invasive method. Moisture content is suspected to play a role in the occurrence of some unwanted phenomena like remobilization of particles on rock walls in caves for example. The hygrothermal conditions are important as they can modify the moisture content of the granular materials on the wall leading to a humidification or at the contrary to a drying of the powder. In this study, optical measurements are used to characterize a wet powder during its drying process. Different steps of this dynamic drying process are modelled and thanks to an adaptation of the existing Melamed's model of scattering of light by a particulate medium, simulation results are compared with instrumental measures. The modelling of such a system allows the estimation of moisture content of a granular material and brings information about the localiza-tion of water in the particulate medium.
\end{abstract}

Keywords Moisture content · Granular material - Optical measurements $\cdot$ Melamed's model $\cdot$ Drying process

\section{Introduction}

Colour is a complex property of materials that can in some cases be considered as a marker of another property more difficult to measure: examples can be found in the textile industry $[1,2]$ or food industry $[3,4]$. In this study, presented in two parts (part I and part II, here), the aim is to show how optical measurements can help to estimate the moisture

Helene Garay

helene.garay@mines-ales.fr

1 C2MA - Ecole des mines d'Alès, 6 avenue de Clavières, 30319 Ales Cedex, France content of granular materials. The optical measurements proposed have the advantage of being non-invasive, an essential property in some fields such as heritage conservation.

In the case of a thick layer of a granular material, colour depends on parameters such as the nature of the particles, their size and shape, and their packing. The presence of water is also a parameter that will change the visual aspect as it can be considered as another constituent of the sample.

In this situation, the main phenomenon is the scattering of light by a concentrated medium i.e. multiple scattering. So models focused on the scattering of light by a single particle such as Mie's theory cannot be used in this study. Another type of model described in the literature considers the sample as successive infinitesimal layers of homogenous material and derives some equations from the radiative transfer equation [5]: the N-flux models [6] are the most precise of these models but the calculations are fastidious. At the opposite extreme, the two-flux model, known as the Kubelka-Munk model $[7,8]$ is the easiest of these models to use but the parameters of this model are the scattering (S) and absorption (K) coefficients of the global system. More recently a model for a monolayer of mono-dispersed particles on a substrate has been proposed [9] and then extended for a monolayer of particles with a particle size distribution [10]. D.J. Dahm et al. consider a sample through a representative layer. With some conditions they showed that it exists a relation between the absorption and reflection of the whole sample and the volume fraction and the fraction of cross section respectively of the representative layer. Melamed's model $[11,12]$ is another type of scattering model, as Melamed calculates the reflected, refracted, absorbed and transmitted parts for single particle and then deduces the behaviour of the global system assuming that the spherical particles are packed in a hexagonal form. Garay et al. [13] showed that this model has some ability to be adapted for another shape of particles: they cal- 
culated the effect of taking ellipsoids of different aspect ratio and showed that the result is closes to the result obtained with spheres. That's another reason to choose this model for this study. The part I of the study shows the experiments and the optical measurements made to track the drying process of a mineral granular sample. The aim is to try to transfer this method to the problem of the control over time of the prehistoric drawings, problem for which the exchange of water between ambient air and paints is crucial. The study presented in this paper is part II of the global study, focusing on the modelling of the drying process. One step in this process needs particular development: the water surrounding the particles (noted particle@water, as in the domain of organic chemistry when a core particle is surrounded with a coating). This situation is modelled considering the ideal case of a core particle surrounded by a shell of water of constant thickness.

\section{Presentation of Melamed's model}

Melamed's model is a theoretical model that describes the scattering of light by a particulate medium in air. The particles are much larger than the incident light wavelength, are spherical, and are arranged in a hexagonal compact manner [11]. The restriction on the size of particles is due to the use of the geometrical optics rules.

Melamed expresses the reflectance $\mathrm{R}$ as a function of $\mathrm{n}$ and $\mathrm{k}$, respectively the real and imaginary part of the complex refractive index, $\mathrm{d}$, the diameter of the particles, $\lambda$ the wavelength of the incident light, and a parameter $\mathrm{x}_{\mathrm{u}}$, specific to this model, which takes into account the arrangement of the particles (Eq. 1). This expression in terms of the physical parameters of the powder is the main interest of this model as it becomes possible to use the model to predict reflectance when certain conditions are changed.

$\mathrm{R}=\mathrm{f}\left(\mathrm{n}, \mathrm{k}, \mathrm{d}, \lambda, \mathrm{x}_{\mathrm{u}}\right)$

The formalism of the model is represented in Fig. 1. For greater clarity, the top layer is separated from the rest of the sample.

Melamed considers unitary radiation incident on the medium. After multiple inter-reflections, a part of this initial radiation emerges upward. The aim of the model is to quantify this part. The function $\mathrm{f}$ of Eq. 1 cannot be written so simply. In fact the variables of this equation are used to calculate other coefficients, specific to the model. Some of these coefficients are calculated for one particle:

- $\mathbf{m}_{\mathbf{e}}$ and $\mathbf{m}_{\mathbf{i}}$ are the coefficients of reflection, respectively external and internal, which describe the way the incident ray interacts with the surface. They depend on both the particle and medium refractive index.

- $\mathbf{M}$ is the coefficient corresponding to the mean attenuation of a ray crossing the particle once (the attenuation

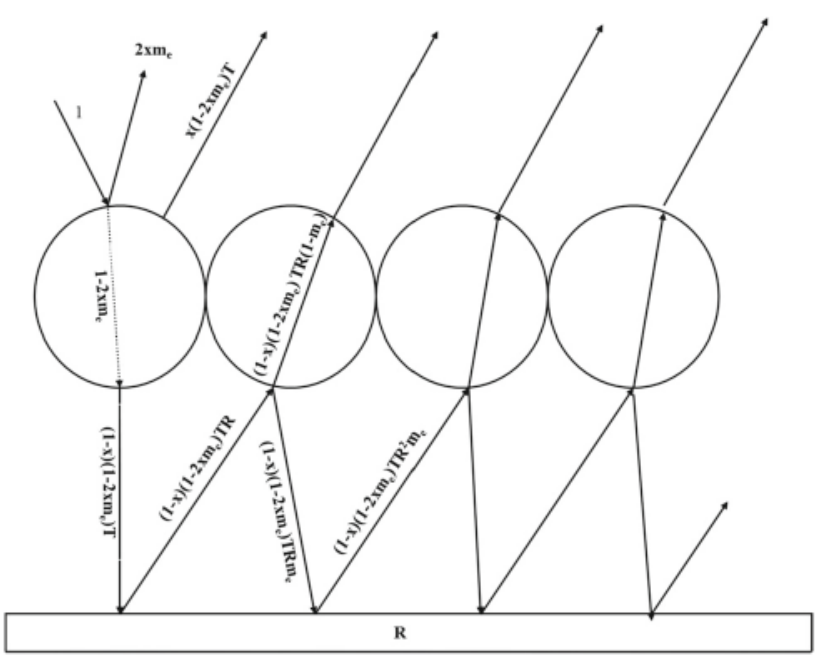

Fig. 1 Formalism of Melamed's model: for greater clarity, the upper layer of particles is separated from the bulk of the particulate medium

will depend on the length of the path in the particle, and therefore on the angle of the initial ray compared with the normal to the particle surface). The coefficient $M$ depends on $\mathrm{k}$, the absorption coefficient and on $\mathrm{d}$, the diameter of the particle.

- T represents the part of the internal radiation that will be "transmitted" by the particle after multiple interreflections (here the term "transmission" takes into account all the rays that leave the particle whatever their direction). It depends on $\mathrm{m}_{\mathrm{i}}$ and $\mathrm{M}$.

A fifth parameter called $\mathbf{x}$ corresponds to the probability that a ray emerges above the sample. It depends on $x_{u}, k, d$ and $\mathrm{T}$. It is this parameter that enables the arrangement of the particles to be taken into account.

The reflectance $\mathrm{R}$, according to Melamed, can be written as:

$R=\frac{1+m_{e}(A+B)+A C-\sqrt{\left(\left(1+m_{e}(A+B)+A C\right)^{2}-4\left(m_{e}+C\right)(A+B)\right)}}{2\left(m_{e}+C\right)}$

A, B and C are auxiliary terms for calculation. They can be expressed as:

$$
\begin{aligned}
& A=2 x m_{e} \\
& B=x\left(1-2 x m_{e}\right) T \\
& C=(1-x)\left(1-m_{e}\right) T
\end{aligned}
$$

The reflectance calculated by the model takes into account all the rays resulting from the interaction of the incident radiation with the medium made up of particles and air. This interaction consists in a specular part (noted $2 \mathrm{xm}_{\mathrm{e}}$ on Fig. 1) 
and in the results of what happens in the bulk of the sample (scattering and absorption).

This means, in order to compare results of model calculations with real measures, that the total signal reflected by the sample has to be collected.

But measuring the reflectance of powders is not as simple as for solid plane materials. The measure will depend on the state of the sample surface, which consists of the upper layer of particles. In the case of powders, it is hard to use an integrating sphere as the powders can pollute the inside coating of the sphere. So, one possibility is to use a spectrogoniophotometer to characterize the angular distribution of the signal, and then, by spatial integration of the signal, to calculate the total emission. That experimental work is detailed in part I of this study.

The aim of this part of the study is to determine how Melamed's model could help to describe the state of a powder during the whole drying process, at least qualitatively, i.e. can this model explain the variation measured by the spectrogoniophotometer thanks to its parameters? The phenomenon of drying is dynamic as the water content varies during the process: water evaporates from the surface and liquid water diffuses from the bulk of the sample to the top of the sample, which is less saturated due to evaporation.

The position of water in a particulate medium will depend on the physic-chemical properties of the particles, the water content, and the position in the sample (near the surface or in the bulk).

\section{Modelling of the drying process}

The experimental part is detailed in part I of this study, to which the interested reader is invited to refer. The sample studied is a paste composed of a yellow ochre and water allowed to dry under ambient conditions.

The drying process of this sample is dynamic but some steps can be identified. They are schematically represented in Fig. 2:

- At the beginning of the observation, the particles are covered by a film of water.

- The water progressively evaporates at the surface and the film is already less smooth.

- Air appears in the bulk of the sample, firstly just under the particles/air interface, and then, due to diffusion of water from the most concentrated medium towards the less concentrated medium, in the bulk.

- "Dry" sample: the sample is considered as dry when its weight is constant under ambient conditions.

Stage 1 can be considered as "particles in water" (as in stage 2) but with a film of water above. So the presence of this film can be modelled as a non-scattering, non-absorbing and smooth medium. This coat will have a specular behaviour.

Stage 3 is the most complicated to model. It's difficult to estimate the thickness of the coat of water around the particles: it's probably not uniform all around the particles, due to the existence of capillary bounds. However, as a first approximation we can consider this coat uniform and thick enough to apply Melamed's model.

The adaptation of Melamed's model for this step is detailed in the following section.

\section{Modelling of the particle@water step}

Looking at the various parameters of the model, the most appropriate parameter for modification is $\mathrm{T}$, corresponding
Fig. 2 Schematic

representation of four stages in the drying process: a Presence of a film of water above the surface; $\mathbf{b}$ the film begins to dry and is not smooth anymore; $\mathbf{c}$ air appears in the bulk of the sample and $\mathbf{d}$ the sample is dry. The classical Melamed's model corresponds to Stage 4 (Fig. 2). Stage 2 is rather simple to model with Melamed's model: only the parts of reflection and refraction at the interface particle/air will be modified and this modification can be estimated by calculating $\mathrm{m}_{\mathrm{e}}$ and $\mathrm{m}_{\mathrm{i}}$, respectively external and internal reflections coefficients, with relative refractive index $\mathrm{n}_{\text {particle }} / \mathrm{n}_{\text {medium }}$

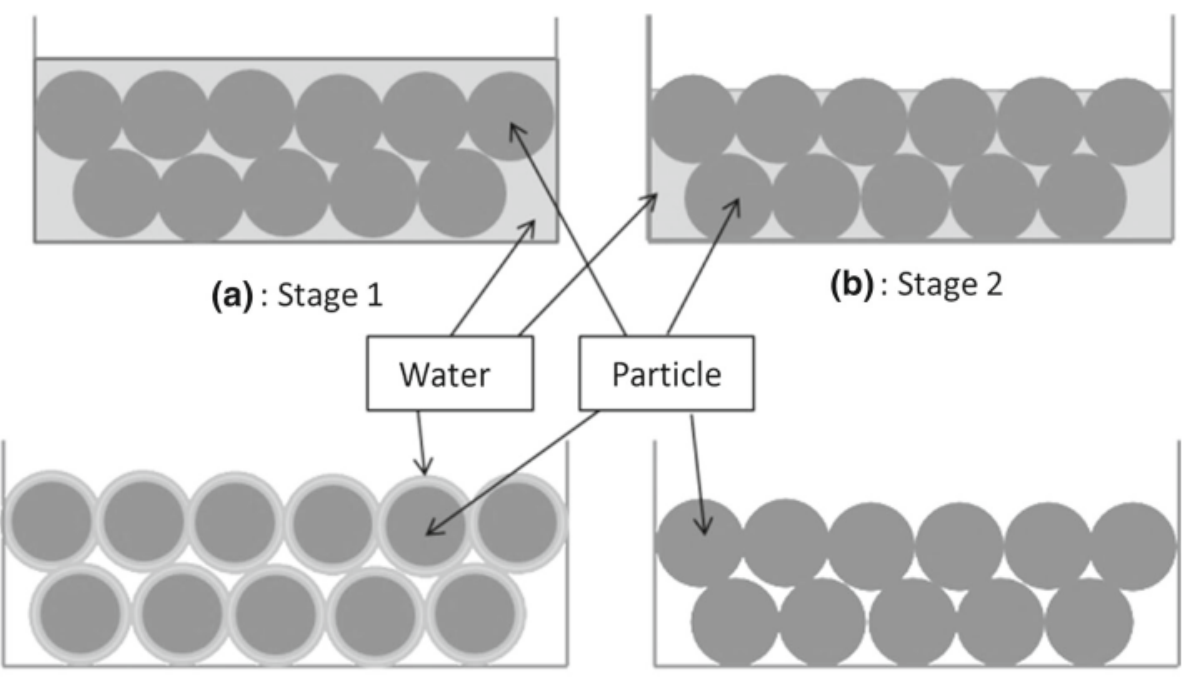

(c) : Stage 3 (d) : Stage 4 
to the part of radiation inside a particle that will go out of that particle after multiple reflections inside it. In the case of the particle@water system, T will be recalculated using the hypotheses that water does not absorb and the coating is thick enough to use the laws of geometrical optics. This is a strong hypothesis because we know that, during the drying process, the thickness of this coat around the particles will not be uniform due to the existence of capillary bounds. Moreover, considering that water doesn't absorb visible light, will not let take into account the variation of thickness of this coat as a parameter. With this type of modelling, it will be impossible to follow all the drying process.

To calculate $\mathrm{T}$, the coefficient $\mathrm{M}$ is needed: in the calculation, the coefficient $\mathrm{M}$ is calculated for one particle as if no water is used.

All the rays that emerge out of the particle@water system have to be taken into account (Fig. 3). In this system, considering geometrical optics, each ray which meets an interface is partly reflected according to Fresnel's law and partly refracted. All the emerging rays come from the multiple reflections inside the water coating. Two coefficients $\mathrm{m}_{\mathrm{i}}$, mean internal reflection coefficients, are defined: firstly $\mathrm{m}_{\mathrm{i} 1}$ at the particle/water interface and secondly, $\mathrm{m}_{\mathrm{i} 2}$, at the water/air interface. Considering $\mathrm{m}_{\mathrm{e} 1}$, the mean external reflection coefficient, the contribution of each ray can then be calculated.

Let us consider an incident ray (noted "a 0 " in Fig. 3). $a_{1}$ is reflected at the surface in the water coat. $a_{0} b_{1}$ is refracted into the particle. The strategy is to focus on the rays coming directly from $\mathrm{a}_{0}$ and calculate their contributions after successive crossings of the particle. The contributions of the other rays coming from interactions of $\mathrm{a}_{0}$ with the water coat will be deduced from the calculation of the contribution of $\mathrm{a}_{0} \mathrm{~b}_{1}$.

The interactions can be written as:

$$
\begin{aligned}
& \mathrm{a}_{1}=\mathrm{m}_{\mathrm{e} 1} \mathrm{a}_{0} \\
& \mathrm{a}_{3}=\mathrm{m}_{\mathrm{i} 2}^{*} \mathrm{a}_{1}=\mathrm{m}_{\mathrm{i} 2}^{*} \mathrm{~m}_{\mathrm{e} 1}^{*} \mathrm{a}_{0} \\
& \mathrm{a}_{2}=\mathrm{a}_{1}-\mathrm{a}_{3}=\mathrm{m}_{\mathrm{e} 1}\left(1-\mathrm{m}_{\mathrm{i} 2}\right) \mathrm{a}_{0} \\
& \mathrm{a}_{5}=\left(\mathrm{m}_{\mathrm{e} 1}\right)^{2} \mathrm{~m}_{\mathrm{i} 2}\left(1-\mathrm{m}_{\mathrm{i} 2}\right) \mathrm{a}_{0} \\
& \mathrm{a}_{8}=\left(\mathrm{m}_{\mathrm{e} 1}\right)^{3}\left(\mathrm{~m}_{\mathrm{i} 2}\right)^{2}\left(1-\mathrm{m}_{\mathrm{i} 2}\right) \mathrm{a}_{0} \text { and so on }
\end{aligned}
$$

The sum of the rays emerging from the interaction of $\mathrm{a}_{1}$ with the particle@water system is

$a_{2}+a_{5}+a_{8}+\cdots$ It can be expressed as a sum of the terms of a geometrical progression in $\mathrm{m}_{\mathrm{e} 1}^{*} \mathrm{~m}_{\mathrm{i} 2}$. With the notations of Fig. 3, this sum can be written as

$$
\sum a_{3 i+2}=\mathrm{a}_{1} \frac{1-m_{i 2}}{1-m_{e 1} m_{i 2}}
$$

The same calculation can be made for each ray similar to $a_{1}\left(a_{0} b_{2} c_{1}\right.$ or $a_{0} b_{4} c_{1}$, etc., on Fig. 3). So, whatever the value of the ray (noted $\mathrm{x}_{1}$ ) coming from the particle and crossing the water coat, its "primary" contribution (i.e. without any further crossing of the particle) can be expressed as

$$
\sum_{j=0}^{\infty} x_{3 j+2}=x_{1} * \frac{1-m_{i 2}}{1-m_{e 1} m_{i 2}}
$$

For $\mathrm{a}_{1}$, Eq. 5 represents the direct contribution for T. But the rays $a_{0} b_{1}, a_{3} b_{1}, a_{6} b_{1}, a_{9} b_{1}, \ldots$ which are refracted into the particle and cross it, have to be considered. The intensities of these rays can be written as:

$$
\begin{aligned}
& \mathrm{a}_{0} \mathrm{~b}_{1}=\mathrm{a}_{0}\left(1-\mathrm{m}_{\mathrm{e} 1}\right) \\
& \mathrm{a}_{3} \mathrm{~b}_{1}=\mathrm{a}_{0}\left(1-\mathrm{m}_{\mathrm{e} 1}\right) \mathrm{m}_{\mathrm{e} 1} \mathrm{~m}_{\mathrm{i} 2} \\
& \mathrm{a}_{6} \mathrm{~b}_{1}=\mathrm{a}_{0}\left(1-\mathrm{m}_{\mathrm{e} 1}\right) \mathrm{m}_{\mathrm{e} 1}^{2} \mathrm{~m}_{\mathrm{i} 2}^{2} \\
& \mathrm{a}_{9} \mathrm{~b}_{1}=\mathrm{a}_{0}\left(1-\mathrm{m}_{\mathrm{e} 1}\right) \mathrm{m}_{\mathrm{e} 1}^{3} \mathrm{~m}_{\mathrm{i} 2}^{3}
\end{aligned}
$$

All these rays will have a similar path in the system so, for each ray, the contribution is a unique function of $a_{0} b_{1}, a_{3} b_{1}$, $a_{6} b_{1}, a_{9} b_{1}$, respectively.

We can note that, whatever $n>0, a_{3 n} b_{1}=a_{3(n-1)}^{*} m_{e 1} m_{i 2}$.

So if $f\left(a_{0} b_{1}\right)$ is the contribution of $a_{0} b_{1}$, the total contribution of $a_{0} b_{1}, a_{3} b_{1}, a_{6} b_{1}, a_{9} b_{1}$ can be written as the sum of this geometrical progression in $\mathrm{m}_{\mathrm{e} 1} \mathrm{~m}_{\mathrm{i} 2}$ i.e.

$\mathrm{T}\left(\mathrm{a}_{0} \mathrm{~b}_{1}, \mathrm{a}_{3} \mathrm{~b}_{1}, \mathrm{a}_{6} \mathrm{~b}_{1}, \mathrm{a}_{9} \mathrm{~b}_{1, \ldots}\right)=\mathrm{f}\left(\mathrm{a}_{0} \mathrm{~b}_{1}\right)^{*}\left(1 /\left(\mathrm{m}_{\mathrm{e} 1} \mathrm{~m}_{\mathrm{i} 2}\right)\right)$

The contribution of $a_{0} b_{1}$ in the same way can be divided in two parts:

- firstly the direct contribution of $\mathrm{a}_{0} \mathrm{~b}_{2} \mathrm{c}_{2}, \mathrm{a}_{0} \mathrm{~b}_{2} \mathrm{c}_{5}, \mathrm{a}_{0} \mathrm{~b}_{2} \mathrm{c}_{8}, \ldots$.

According to Eq. 6, this contribution can be written as

$\sum a_{0} b_{2} c_{3 j+2}=a_{0} b_{2} c_{1} \frac{1-m_{i 2}}{1-m_{e 1} m_{i 2}}$

- And secondly the contribution of $a_{0} b_{3}, a_{0} b_{3} c_{3}, a_{0} b_{3} c_{6}$, $a_{0} b_{3} c_{9}$, refracted in the particle can be written, as in Eq. 8:

$$
\begin{aligned}
& \mathrm{T}\left(\mathrm{a}_{0} \mathrm{~b}_{3}, \mathrm{a}_{0} \mathrm{~b}_{3} \mathrm{c}_{3}, \mathrm{a}_{0} \mathrm{~b}_{3} \mathrm{c}_{6}, \mathrm{a}_{0} \mathrm{~b}_{3} \mathrm{c}_{9}, \ldots\right) \\
& \quad=\mathrm{f}\left(\mathrm{a}_{0} \mathrm{~b}_{3}\right)^{*}\left(1 /\left(\mathrm{m}_{\mathrm{e} 1} \mathrm{~m}_{\mathrm{i} 2}\right)\right)
\end{aligned}
$$

This calculation, focusing on $\mathrm{a}_{0} \mathrm{~b}_{5}$, and then on $\mathrm{a}_{0} \mathrm{~b}_{7}$ and $\ldots \mathrm{a}_{0} \mathrm{~b}_{2 \mathrm{n}+1}$ can be continued.

So, for the calculation of the coefficient $\mathrm{T}$, all the contributions have to be taken into account,

$$
T=a_{1} \frac{1-m_{i 2}}{1-m_{e 1} m_{i 2}}+\frac{1}{1-m_{e 1} m_{i 2}} f\left(a_{0} b_{1}\right)
$$




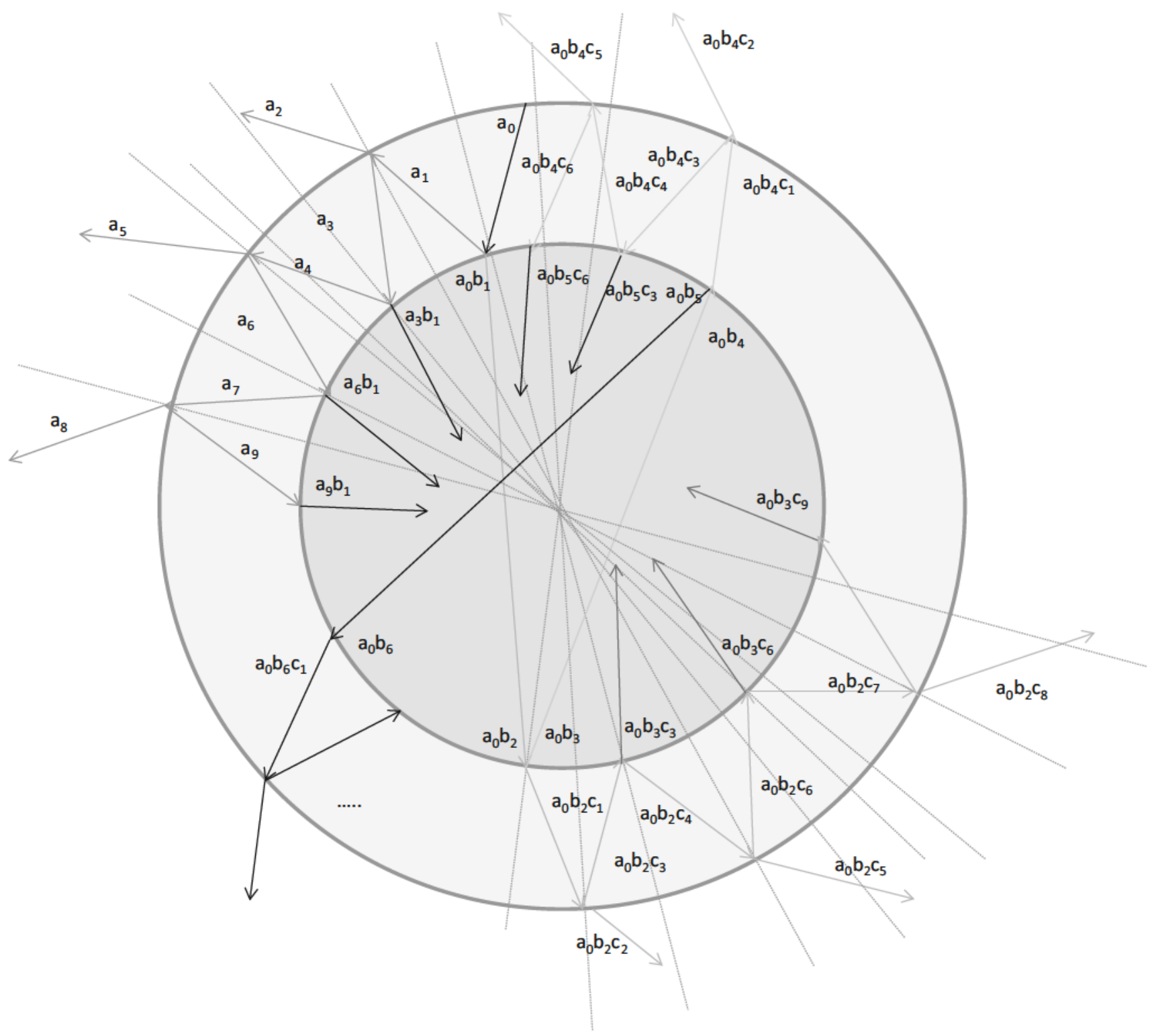

Fig. 3 Path of an initial ray $\mathrm{a}_{0}$ inside the particle@water system

$$
\begin{aligned}
T= & a_{1} \frac{1-m_{i 2}}{1-m_{e 1} m_{i 2}}+\frac{1}{1-m_{e 1} m_{i 2}} \\
& {\left[a_{0} b_{2} c_{1} \frac{1-m_{i 2}}{1-m_{e 1} m_{i 2}}+\frac{1}{1-m_{e 1} m_{i 2}} f\left(a_{0} b_{3}\right)\right] }
\end{aligned}
$$

Continuing the calculation of $\left(a_{0} b_{5}\right)$ :

$$
\begin{aligned}
T= & a_{1} \frac{1-m_{i 2}}{1-m_{e 1} m_{i 2}}+\frac{1}{1-m_{e 1} m_{i 2}} a_{0} b_{2} c_{1} \frac{1-m_{i 2}}{1-m_{e 1} m_{i 2}} \\
& \left.+\frac{1}{1-m_{e 1} m_{i 2}}\left[a_{0} b_{4} c_{1} \frac{1-m_{i 2}}{1-m_{e 1} m_{i 2}}+\frac{1}{1-m_{e 1} m_{i 2}} f\left(a_{0} b_{5}\right)\right]\right\}
\end{aligned}
$$

Continuing this calculation,

$$
\begin{aligned}
& T(n)=a_{1} \frac{1-m_{i 2}}{1-m_{e 1} m_{i 2}}+\frac{1-m_{i 2}}{\left(1-m_{e 1} m_{i 2}\right)^{2}} a_{0} b_{2} c_{1} \\
& \quad+\frac{1-m_{i 2}}{\left(1-m_{e 1} m_{i 2}\right)^{3}} a_{0} b_{4} c_{1}+\frac{1-m_{i 2}}{\left(1-m_{e 1} m_{i 2}\right)^{4}} a_{0} b_{6} c_{1}+\cdots \\
& \quad+\frac{1-m_{i 2}}{\left(1-m_{e 1} m_{i 2}\right)^{n+1}} a_{0} b_{2 n} c_{1}+f\left(a_{0} b_{2 n+1}\right) \frac{1}{\left(1-m_{e 1} m_{i 2}\right)^{n+1}}
\end{aligned}
$$$$
\lim _{n \rightarrow \infty} T(n)=m_{e 1} a_{0} \frac{1-m_{i 2}}{1-m_{e 1} m_{i 2}}+M\left(1-m_{e 1}\right)
$$$$
\left(1-m_{i 1}\right)\left(1-m_{i 2}\right) a_{0} \sum_{n} \frac{m_{i 1}^{n-1} M^{n}}{\left(1-m_{e 1} m_{i 2}\right)^{n+1}}
$$

as $\lim _{n \rightarrow \infty} f\left(a_{0} b_{2 n+1}\right) \frac{1}{\left(1-m_{e} 1 m_{i 2}\right)^{n+1}}=0$

because $0<m_{e 1} m_{i 2}<1$ and $f\left(a_{0} b_{2 n+1}\right)$ is a finite number. 


\section{Calculation and comparison with measures}

The modelling method described in this study has been used to calculate reflectance of a sample whose characteristics are:

Median particle size: $\mathrm{d}=5 \mu \mathrm{m}$.

Refractive index: $\mathrm{n}=2.3$ [14]

These characteristics are those of the ochre sample used in the experiments described in Part I of this study. In this section, the measurements analysed in Part I are compared with the modelling results.

A wavelength is selected: $620 \mathrm{~nm}$. As it corresponds to a zone of the spectrum with high reflectance for the sam$\mathrm{ple}$, the signal/noise ratio is maximized. The estimation of $\mathrm{k}$, the absorption coefficient, is difficult, especially for granular materials. We know that reflectance is a monotone decreasing function of the absorption coefficient $\mathrm{k}$. So it's possible to solve numerically - by dichotomy - the Melamed's equation in a reverse form (i.e. $\mathrm{k}$ in function of $\mathrm{R}, \mathrm{n}, \mathrm{d}$, $\mathrm{xu}$ and $\lambda)$. In that case, $R$ is the reflectance value measured for the sample in the dry state [15]. The value of $k$ obtained thanks to this calculation is then used to model the other steps in the drying process. This is possible because the modelling of the other steps of the drying process is made with the same particles with, either water, or a mixture of water and air in the inter-particular space so we can consider the same value of $\mathrm{k}$ for all the particles.

At $620 \mathrm{~nm}$, the measured value for the reflectance of the dry sample is: $R=0.53$

Solving numerically Eq. 2 in a reverse form gives $\mathrm{k}=$ $6.51 .10^{-4}$

Then knowing $\mathrm{k}, \mathrm{n}, \mathrm{d}, \mathrm{x}_{\mathrm{u}}$, and $\lambda$, the reflectance, according to Eq. 2 with some adaptations described in the Sects. 2 and 3 of this study, is calculated. The results of these calculations are presented in Table 1.

The value of the reflectance at $620 \mathrm{~nm}$ during the drying process is extracted from the measurement data set and presented in Fig. 4, where the values from Table 1 are added on the curve. It's given in terms of moisture content expressed in dry basis (w d.b.):

$w_{d . b .}=100 \frac{m_{w}-m_{d w}}{m_{d}}$

Where $m_{w}$ is the weight of the humid powder and $m_{d}$ is the weight of the dry powder.

Table 1 Calculation of reflectance

\begin{tabular}{ll}
\hline Drying process steps & Calculated R \\
\hline Particles in water with a film of water upside & 0.679 \\
Particles in water without the film upside & 0.613 \\
Particles@ water system & 0.353 \\
\hline \hline
\end{tabular}

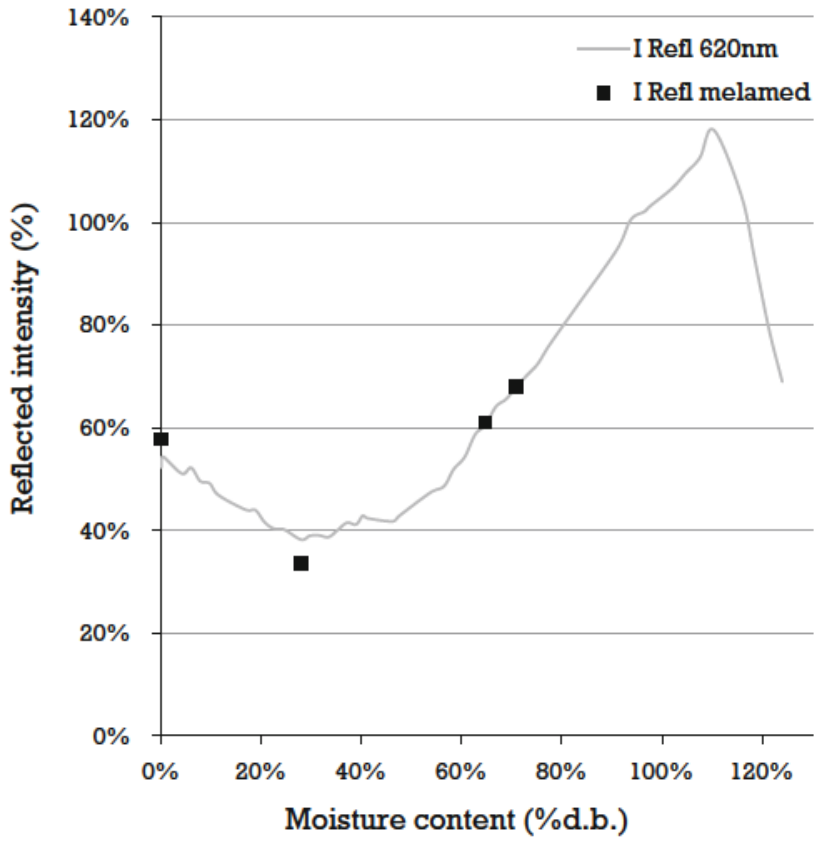

Fig. 4 Measured reflectance at $620 \mathrm{~nm}$ along the drying process (curve) and calculated values for some particular steps (squares). d.b. means "dry basis" as explained before

The calculated values are coherent with the measurements: there is a moment in the drying process which corresponds to the calculated reflectance values and this moment is in accordance with our description of the system made to define the modelling hypothesis.

The drying process is dynamic and our model focuses on specifically identified situations. Nevertheless, this model gives some information about the water content of a granular sample without any invasive nor destructive experiment.

In particular, the minimum of the curve (at $30 \%$ of moisture d.b.) can be modelled, i.e. the calculated values follow the trend of the measured curve even if we know that our system is probably never the same as the one we model. This study shows the flexibility of the Melamed's model which, thanks to its philosophy based on calculations made concerning the particles, can be adapted for more complicated systems than ideal spheres such as the particle@water system considered in this part of the study.

\section{Conclusion}

The study presented in this paper shows that Melamed's model for the scattering of light by a particulate medium presents a real flexibility as it gives the possibility to modify some parameters of the model to enable us to take into account the water content of a granular material.

Working on various coefficients of the model, it can calculate the reflectance of particles in other media such as water, 
and calculate the reflectance of particles surrounded by a coating of water in air.

The drying process of such a granular system is dynamic and this modelling with Melamed's model focuses on some well-defined situations. The modelling process follows the variations in reflectance with the water content in the sample. In particular, it can identify the minimum point of the curve of reflectance at $620 \mathrm{~nm}$ versus moisture content. This result is of interest for example to give information about complex media such as powders applied to a rocky surface. Hygrothermal conditions can modify the water content of the powder and the results of this study can help to have a better knowledge of the water content and its localization in such a sample. This better knowledge will be helpful for studying such systems in locations which are difficult to access, meaning that only portable instruments can be used.

\section{References}

1. Cao, J., Wu, X.: Partial correlation analysis of color reflectance of dynamic moisture transfer of fabric and static physical indicators of fabric. Appl. Mech. Mater. 170-173, 2987-2990 (2012)

2. Dalton, P.M., Nobbs, J.H., Rennell, R.W.: The influence of moisture content on the colour appearance of dyed textile materials. I. Dyeing methods and reflectance measurements on wool. J. Soc. Dyers Colour. 11(9), 285-287 (1995)

3. Figiel, A.: Drying kinetics and quality of beetroots dehydrated by combination of convective and vacuum-microwave methods. J. Food Eng. 98(4), 461-470 (2010)
4. Romano, G., Argyropoulos, D., Nagle, M., Khan, M.T., Müller, J.: Combination of digital images and laser light to predict moisture content and color of bell pepper simultaneously during drying. J. Food Eng. 109(3), 438-448 (2012)

5. Chandrasekhar, S.: Radiative Transfer. Dover, New-york (1960)

6. Maheu, B., Letoulouzan, J.N., Gouesbet, G.: Four-flux models to solve the scattering transfer equation in terms of Lorenz-Mie parameters. Appl. Opt. 23(19), 3353 (1984)

7. Kubelka, P.: New contributions to the optics of intensely lightscattering materials, part I. J. Opt. Soc. Am. 38, 448-457 (1948)

8. Kubelka, P., Munk, F.: Ein Beitrag zur Optik der Farbanstriche. Z. Tech. Phys. 12, 593-601 (1931)

9. García-Valenzuela, A., Gutiérrez-Reyes, E., Barrera, R.G.: Multiple-scattering model for the coherent reflection and transmission of light from a disordered monolayer of particles. J. Opt. Soc. Am. A Opt. Image Sci. Vis. 29(6), 1161-1179 (2012)

10. Vázquez-Estrada, O., García-Valenzuela, A.: Optical reflectivity of a disordered monolayer of highly scattering particles: coherent scattering model versus experiment. J. Opt. Soc. Am. A Opt. Image Sci. Vis. 31(4), 745-754 (2014)

11. Melamed, N.: Optical properties of powders. Part I. Optical absorption coefficients and the absolute value of the diffuse reflectance. J. Appl. Phys. 34(3), 560 (1963)

12. Mandelis, A., Boroumand, F., van den Bergh, H.: Quantitative diffuse reflectance and transmittance spectroscopy of loosely packed powders. Spectrochim. Acta A Mol. Spectrosc. 47(7), 943-971 (1991)

13. Garay, H., Eterradossi, O., Benhassaine, A.: Should Melamed's spherical model of size-colour dependence in powders be adapted to non-spheric particles? Powder Technol. 156(1), 8-18 (2005)

14. http://www.naturalpigments.com/yellow-ocher-jcl.html. Access ed 12 Nov 2015

15. Garay, H., Eterradossi, O., Benhassaine, A.: Predicting changes in the color of powders: does Melamed's model fit to real industrial powders? Color Res. Appl. 29(6), 413-419 (2004) 